\title{
INSPIRAÇÃO, COMUNICAÇÃO E EMPATIA COMO CARACTERÍSTICAS ESSENCIAIS À FORMAÇÃO DO MÉDICO HUMANISTA: INOVANDO ATRAVÉS DE UNIVERSIDADES CRIATIVAS
}

\author{
INSPIRATION, COMMUNICATION AND EMPATHY AS ESSENTIAL \\ CHARACTERISTICS TO THE TRAINING OF THE HUMANIST PHYSICIAN: \\ INNOVATING THROUGH CREATIVE UNIVERSITIES
}

\author{
DE OLIVEIRA, Carolina Maciel ${ }^{1}$ \\ SILVA, Ana Flávia Assis ${ }^{2}$ \\ BERTOL, Sônia Regina Schena ${ }^{3}$ \\ BRITO, Rebeca Simões ${ }^{4}$ \\ MOURÃO JÚNIOR, Carlos Alberto ${ }^{5}$ \\ DE OLIVEIRA, Camila Maciel ${ }^{6}$
}

\section{Resumo}

Contribuir para o explorar de novas ideias e possibilidades, por meio de metodologias que possam inspirar, instigar a criatividade e estimular o pensamento inovador de estudantes do curso de Medicina, é um dos objetivos do programa "Little Hearts Changing Lives" (LHCL). Projetos de extensão com esta característica proporcionam, ainda, potencial para o desenvolvimento de competências como empatia e comunicação, essenciais para a formação do médico humanista. A metodologia que compõe o programa LHCL oferece estratégias para que os discentes sejam envolvidos em atividades criativas de aprendizagem. Desta forma, relatamos a experiência

\footnotetext{
${ }^{1}$ Psicóloga formada pela PUC-SP. Baependi, MG, Brasil e-mail: carolinamaciel@outlook.com ${ }^{2}$ Universidade Federal de Juiz de Fora - UFJF. Juiz de Fora, MG, Brasil. https://orcid.org/0000-0002-01072933 e-mail: anafas2010@gmail.com

${ }^{3}$ Universidade Federal de Passo Fundo - UFP. Passo Fundo, RS, Brasil. https://orcid.org/0000-0003-33158587 e-mail: sobertol@upf.br

${ }^{4}$ Cultural Agents Initiative at Harvard University. Boston, Massachusetts, USA. https://orcid.org/0000-00018833-6999 e-mail: rebecabrito@culturalagents.org

${ }^{5}$ Universidade Federal de Juiz de Fora - UFJF / Departamento de Fisiologia. Juiz de Fora, MG, Brasil. https://orcid.org/0000-0001-7199-5365 e-mail: camouraojr@gmail.com

${ }^{6}$ Massachusetts Institute of Technology - MIT / Institute for Medical Engineering and Science. Cambridge, Massachusets, USA. Universidade Federal do Paraná - UFPR. Curitiba, PR, Brasil. https://orcid.org/00000001-6823-7395 e-mail: cmaciel@mit.edu
} 
DOI: $10.12957 / \mathrm{e}-\mathrm{mosaicos} .2021 .45222$

extramuros de discentes do quarto período do curso de Medicina da Universidade Federal de Juiz de Fora (UFJF), em que o conhecimento adquirido na disciplina de Fisiologia Cardiovascular fora aplicado à comunidade escolar com o intuito de incentivar a mudança de comportamento acerca de hábitos de vida saudáveis.

Palavras-chave: Ensino médico. Projetos comunitários. Promoção da saúde. Aprendizagem criativa. Teoria do aprendizado social.

\section{ABSTRACT}

Contributing to the exploration of new ideas and possibilities, through methodologies that can inspire, instigate creativity and stimulate the innovative thinking of medical students, is one of the objectives of the Little Hearts Changing Lives (LHCL) program. Extension projects with this characteristic also provide potential for the development of skills such as empathy and communication, essential for the training of the humanist physician. The methodology that makes up the LHCL program offers strategies for students to be involved in creative learning activities. In this way, we report the extramural experience of students from the fourth period of Medicine course at the Federal University of Juiz de Fora (UFJF), where the knowledge acquired in the discipline of Cardiovascular Physiology was applied to the school community in order to encourage the change of behavior about healthy lifestyle habits.

KEYWORDS: Teaching medical education. Community projects. Health promotion. Cardiovascular physiology. Social learning theory.

\section{INTRODUÇÃO}

\section{InSPIRAÇÃo ATRAVÉS DE UNIVERSIDADES CRIATIVAS}

Recentemente, e cada vez mais, a busca por abordagens e desenhos curriculares diferenciados que permitam a formação de profissionais preparados para o mundo real, faz-se evidente. Na China, por exemplo, observou-se que alunos denominados " $A$ ", apesar de obterem os escores mais altos no que se refere a notas, não demonstravam aptidão ou habilidade como a criatividade ou o potencial para inovação, necessários para a obtenção de sucesso diante das demandas da sociedade atual. (RESNICK, 2007; RESNICK, 2017) Esta constatação resultou, então, no incentivo ao desenvolvimento de competências específicas, como a resolução de problemas próprios e/ou situações reais, ao invés da perpetuação do estímulo ao exercício de questões em livros texto. Aqueles estudantes,

INSPIRAÇÃO, COMUNICAÇÃO E EMPATIA COMO CARACTERÍSTICAS ESSENCIAIS 
DOI: $10.12957 / \mathrm{e}-\mathrm{mosaicos} .2021 .45222$

definidos como alunos " $X$ ", potencialmente apresentariam características como sugestões de ideias inovadoras e direcionamentos criativos (RESNICK, 2007; RESNICK, 2014; RESNICK, 2017).

A preocupação com a formação de indivíduos que estejam preparados para o enfrentamento de situações inesperadas - em que as mesmas possam ser resolvidas de forma criativa e/ou inusitada - é fundamentada em teorias que sugerem, por exemplo, que dois terços dos estudantes de hoje trabalharão em atividades que ainda não foram inventadas (RUSK et al., 2009; RESNICK, 2017). A necessidade do estímulo ao pensamento criativo, por sua vez, não se limitaria ao ambiente de trabalho, mas alcançaria situações novas que serão apresentadas nas próximas décadas, no dia a dia de cada indivíduo.

Explorar, então, novas ideias e possibilidades, por meio de metodologias que possam oferecer inspiração, instigar a criatividade e estimular a inovação, contribui para a formação de indivíduos que possam se beneficiar quando do surgimento de desafios e de oportunidades num futuro próximo. Com este objetivo, a metodologia que compõe o programa "Little Hearts Changing Lives" propõe atividades a serem elaboradas e construídas pelos próprios discentes, oferecendo estratégias para que os mesmos sejam envolvidos em experiências criativas de aprendizagem. Desta forma, desde 2014, cultivamos o conceito de Universidades Criativas, em que os discentes, em duplas ou em grupos, são inspirados a criarem os próprios projetos, incluindo poesia rimada, cenários, fantasias e personagens para o teatro de fantoches, além de atividades pedagógicas a serem oferecidas em escolas de municípios brasileiros. Os discentes são, ainda, estimulados a capacitarem colegas recém-chegados ao programa através do compartilhamento do conhecimento e de experiências; coordenação da logística e agenda de atividades; e, confecção dos artigos para publicação (BERMUDEZ et al, 2017; COELHO et al, 2017; GONÇALVES et al, 2016; MADUREIRA et al, 2016; GONÇALVES et al, 2017; SOARES et al, 2017; SOARES et al, 2017; BRITO et al, 2019). Contempla-se, assim, os quatro pilares compreendidos na conceituação de Aprendizagem Criativa: paixão (passion), trabalho em pares ou em grupo (peers), projetos reais (project), de forma lúdica (play) (RESNICK, 2007; RESNICK, 2014; RESNICK, 2017).

\section{ESTRATÉGIAS PARA A TRANSMISSÃO DE INFORMAÇÃO}

\section{UNIVERSIDADES}

Ferramentas interessantes têm sido utilizadas por docentes dos cursos de Medicina em algumas instituições. Metodologias ativas presenciais ou o ensino híbrido, a cada dia,

INSPIRAÇÃO, COMUNICAÇÃO E EMPATIA COMO CARACTERÍSTICAS ESSENCIAIS 392 À FORMAÇÃO DO MÉDICO HUMANISTA: INOVANDO ATRAVÉS DE UNIVERSIDADES 
DOI: $10.12957 / \mathrm{e}-\mathrm{mosaicos} .2021 .45222$

conquistam mais tempo e espaço nos currículos universitários. Um dos métodos de estudo para fixação do conteúdo de Fisiologia Cardiovascular consiste na dinâmica $A B E$, um conjunto de atividades que tem como objetivo um melhor aprendizado dentro da própria faculdade, diferente dos Projetos de Extensão, que se exteriorizam. A dinâmica ABE é composta por três etapas: a "preparação", em que o aluno estuda individualmente por referências sugeridas pelo professor; a "garantia da preparação", por meio de uma avaliação pequena realizada individualmente ou em grupo, em sala de aula, a qual é sucedida por discussão; e, a "aplicação dos conceitos", por meio da execução de tarefas relacionadas ao tema abordado, em grupo, podendo ser realizada em sala de aula ou junto à comunidade (KRUG et al., 2016).

Por outro lado, atividades que prezem pelo desenvolvimento do aluno como um ser complexo são importantes para o crescimento do mesmo como profissional. É fundamental considerar habilidades como a comunicação e a empatia para a formação de um médico mais humanista. A comunicação adequada aliada à abordagem acertada tanto garante 0 apoio ao paciente e/ou familiar diante de notícias desagradáveis quanto contribui para a construção do vínculo médico-paciente ou, ainda, o estímulo à adesão ao tratamento (SOMBRA NETO et al, 2017).

\section{ENSINo Básico E Fundamental}

Incorpora-se, ainda, a este estudo a visão da Teoria do Aprendizado Social, segundo a qual as pessoas não nascem com repertórios de comportamento, mas podem aprendê-los tanto por experiência direta quanto por observação. A importância da comunicação adequada como estratégia para a aquisição e a manutenção de conhecimentos acerca de hábitos de vida saudáveis é o que nos explicita a Teoria do Aprendizado Social do canadense Albert Bandura. Esta é uma das teorias que mais comumente vêm sendo utilizadas ao definir como criar mensagens para motivar mudanças de comportamento relativas à saúde. Para ele, nem as pessoas estão totalmente condicionadas pelo seu meio, nem as forças do ambiente agem sobre as pessoas como se estas fossem desprovidas de poder; na sua concepção, pessoas e ambientes determinamse mutuamente um ao outro.

"A proposta da Teoria do Aprendizado Social é a explanação do comportamento humano em termos de uma contínua e recíproca interação entre determinantes cognitivos, comportamentais e ambientais." [tradução nossa] (BANDURA, 1977, p. vii).

Bandura relata três características em sua teoria: a ênfase aos papéis proeminentes desempenhados por processos vicários, simbólicos e autorregulatórios no funcionamento psicológico. No que se refere aos processos vicários, afirma que há o reconhecimento de que pensamento, afeto e comportamento humanos podem ser influenciados pela

INSPIRAÇÃO, COMUNICAÇÃO E EMPATIA COMO CARACTERÍSTICAS ESSENCIAIS 393 À FORMAÇÃO DO MÉDICO HUMANISTA: INOVANDO ATRAVÉS DE UNIVERSIDADES 
DOI: $10.12957 / \mathrm{e}-\mathrm{mosaicos} .2021 .45222$

observação; quanto aos processos simbólicos, é fundamental a constatação de que a capacidade de usar símbolos habilita os homens a representar eventos, a analisar sua própria experiência, a se comunicar com outros, a criar e a imaginar; e sobre os processos autorregulatórios, afirma que as pessoas não são simplesmente seres que reagem ao mundo externo, mas que selecionam, organizam e transformam o estímulo que dele provém. Desta forma, "quando diversas condições ambientais produzem variações correspondentes no comportamento, a causa íntima postulada não pode ser menos complexa do que seus efeitos." [tradução nossa] (BANDURA, 1977, p. 3).

Na visão sobre o comportamento humano e suas reações, evidencia-se o conceito de "interação", que, para a Teoria do Aprendizado Social, é um processo no qual comportamento, outros fatores pessoais e fatores ambientais interagem reciprocamente. São fatores independentes, mas que exercem influência uns sobre os outros. Além disso, determinantes internos também devem ser levados em conta no estudo do comportamento, já que o aprendizado e a cognição têm demonstrado grande eficácia ao aprender e reter um comportamento. Na visão da Teoria do Aprendizado Social, pessoas nem são dirigidas por forças íntimas nem atacadas por estímulos de seu ambiente; ao contrário, há uma contínua interação recíproca entre determinantes pessoais e ambientais. A partir desta suposição, é que os processos vicários, simbólicos e autorregulatórios assumem um papel proeminente.

No contexto em que repertórios de comportamento são passíveis de aprendizagem - tanto por experiência direta quanto por observação - encontramos embasamento para o que hoje consideramos como sucesso: o programa "Little Hearts Changing Lives". Desta forma, relatamos neste artigo a experiência extramuros de discentes do quarto período do curso de Medicina da Universidade Federal de Juiz de Fora (UFJF), em que conhecimentos adquiridos durante o curso de Fisiologia Cardiovascular fora aplicado à comunidade escolar, em formato de projeto de extensão, com o intuito de incentivar mudanças de comportamento acerca de hábitos de vida saudáveis para crianças de 6 a 12 anos de idade.

\section{Metodologia}

A metodologia utilizada foi desenvolvida em conjunto, entre discentes e docentes do curso de Medicina da Universidade Federal de Juiz de Fora (UFJF), Departamento de Fisiologia, tendo como espinha dorsal o conceito STEAM (Science, Technology, Engineering, Art and Math)

Após iniciar as apresentações de peças de teatro "O estômago Apaixonado por Doces e o Coração Partido" no município de Baependi, Minas Gerais, o projeto recebeu o nome de "Coraçõezinhos de Baependi". Estas atividades tiveram o intuito de resgatar

INSPIRAÇÃO, COMUNICAÇÃO E EMPATIA COMO CARACTERÍSTICAS ESSENCIAIS 394 À FORMAÇÃO DO MÉDICO HUMANISTA: INOVANDO ATRAVÉS DE UNIVERSIDADES 
DOI: $10.12957 /$ e-mosaicos.2021.45222

aspectos da cultura popular por meio da paródia, além de transmitir conhecimentos adquiridos durante a disciplina de Fisiologia Cardiovascular.

Neste relato de experiência, abordamos a primeira peça apresentada em Baependi, "O Estômago Apaixonado por Doces e o Coração Partido". Esta peça foi realizada em escolas da rede pública e privada do município. Foi apresentada nas seguintes escolas da rede pública: Escolas Municipal Wenceslau Braz, Escola Estadual Senador Alfredo Catão e Escola Municipal Raquel C. Gonçalves. Na rede privada de ensino, para a Escola Santo Inácio. As peças foram realizadas em quatro dias de apresentação, nos turnos da manhã e tarde, sendo cada apresentação de aproximadamente trinta minutos, incluindo a conversa introdutória antes da peça e a roda de paródia após a mesma.

\section{Atividades ANTES DA PEÇA DE TEATRO}

Antes da peça, é realizado um bate-papo com as crianças com o intuito de discutir novas palavras e conceitos que serão utilizados durante o teatro, caso estes não sejam de conhecimento dos alunos. Este momento é chamado de "bate-papo sobre as novas palavras: aprendendo a linguagem científica" e é adaptado ao vocabulário do público em questão, esclarecendo eventuais dúvidas para alcançar o objetivo do projeto. Algumas das palavras e alguns dos conceitos serão ilustrados na tabela 1. Essa atividade é realizada por meio de perguntas como: "Vocês conhecem a palavra saltitar? O que ela significa para vocês? Vocês acham que se parece com pular?", com o objetivo de instigar a curiosidade dos alunos e dar a eles autonomia para participarem do projeto, iniciando assim o vínculo com o público para um melhor resultado. Na peça "O Estômago Apaixonado por Doces e o Coração Partido", o vocabulário sugerido foi estômago, saltitar, ritmo, salivar, digestão, enzimas, glicose, gordura, proteína.

\section{O TEATRO DE FANTOCHES}

O teatro é feito sob a proposta de fantoches, confeccionados pela própria equipe do projeto, sendo de grande relevância a opção por materiais reutilizáveis e que ficarão guardados para as próximas apresentações e equipes. A cortina que compõe o cenário também é feita pela equipe. Os materiais contêm cores alegres, chamando a atenção das crianças para a encenação. As falas das personagens são curtas e compostas por rimas simples, buscando atrair e divertir o público infantil. Ao mesmo tempo em que se transmite o ensinamento sobre uma questão de saúde, convidamos as próprias crianças e recitarem algumas estrofes, como abaixo:

INSPIRAÇÃO, COMUNICAÇÃO E EMPATIA COMO CARACTERÍSTICAS ESSENCIAIS À FORMAÇÃO DO MÉDICO HUMANISTA: INOVANDO ATRAVÉS DE UNIVERSIDADES 
DOI: $10.12957 /$ e-mosaicos.2021.45222

"Coração e Estômago, prometo!

A minha parte fazer.

Prestar bem atenção!

Para isto tudo aprender!

Legumes, verduras e frutas!

Prometo que vou comer!

Exercício físico também

Vou brincar e correr."

Fonte: Metodologia aprendendo a aprender: como aprender brincando.

Autora: Prof. Dra. Camila Maciel de Oliveira

\section{Após A PEÇA DE TEATRO}

Após a peça, os alunos são motivados a refletir sobre o que eles aprenderam até ali, momento conhecido como "Ampliar os conceitos: como explorar o assunto sob aspectos interdisciplinares". Nesta hora, é propiciado um momento de interação, no qual os alunos que quiserem se manifestar sobre os princípios relevantes da peça e levantar questões sobre o que não ficou claro, são convidados a participar, contando também histórias do cotidiano. Neste relato, foram fatos relacionados a familiares cardiopatas, alimentação saudável, consumo abusivo de doces, dentre outros assuntos.

Além do momento de interação, é realizado um momento de atividades lúdicas, como cantiga de roda sob a forma de paródias.

\section{RESULtAdOS E Discussão}

Em todo o mundo, a maioria das escolas prioriza o ensino em que os discentes sejam capazes de repetir ações, decorar tópicos e escolher a resposta correta em avaliações tradicionais, construindo então alunos " $A$ ". Isto é conseguido em detrimento ao desenvolvimento de estratégias que possam potencializar o surgimento de ideias e objetivos singulares em cada indivíduo, o que seria fundamental à formação do pensador "X" (RESNICK, 2007; RESNICK, 2017). O reconhecimento da necessidade de alteração tanto de objetivos quanto de aparato metodológico utilizado pelo sistema educacional tem ocorrido de forma gradual, abrangendo desde o ensino básico aos vários cursos de graduação. A publicação da Base Nacional Comum Curricular (BNCC, 2018) - em que dez competências socioemocionais são contempladas - reforça a necessidade da formação de

INSPIRAÇÃO, COMUNICAÇÃO E EMPATIA COMO CARACTERÍSTICAS ESSENCIAIS 
DOI: $10.12957 / \mathrm{e}-\mathrm{mosaicos} .2021 .45222$

indivíduos, na verdade, cidadãos, com características compatíveis com o aluno "X (AUR, 2018).

Desta forma, a coluna dorsal deste projeto de extensão foi a proposta de realização de atividades lúdicas, perfazendo o conceito STEAM, como o teatro interativo de fantoches e atividades musicais como paródias, tangenciando temas relacionados à saúde e a hábitos de vida saudáveis. A utilização do programa LHCL visava à abertura de canais de comunicação e à facilitação do aprendizado, em escolas do Ensino Básico. A oportunidade de aprendizagem é ofertada sob dois principais pontos de vista: os discentes por um ângulo e crianças e pré-adolescentes por outro. $E$, isto pôde ser consolidado de forma ampla a partir do vínculo estabelecido entre a equipe do programa (discentes e docentes) e professores e alunos das escolas em que atuaram.

Assim, em fevereiro de 2015, 675 alunos (6 a 12 anos), de quatro escolas do município de Baependi, participaram de atividades propostas pelos discentes integrantes do programa LHCL. Peças de teatro, utilizando fantoches e poesia rimada, foram apresentadas nas escolas Escola Municipal Wenceslau Braz $(n=270$ alunos), Escola Estadual Senador Alfredo Catão ( $n=225$ alunos), Escola Municipal Raquel C. Gonçalves $(n=130$ alunos), Escola Santo Inácio $(n=50$ alunos). Este programa foi apelidado inicialmente de "Coraçõezinhos de Baependi" por ter iniciado sua atuação no município de mesmo nome, em Minas Gerais. A cidade fora escolhida por residir a primeira coorte para estudo genético de doenças cardiovasculares e outras doenças crônicas não transmissíveis no Brasil (DE OLIVEIRA et al, 2008). Atualmente, o programa é composto por docentes e discentes da Universidade Federal de Juiz de Fora (UFJF), Universidade Federal do Paraná (UFPR) e Unipac-JF, sendo responsável por intervenções relacionadas à promoção da saúde neste município, tanto na zona urbana quanto na zona rural desta cidade. A expansão do programa foi realizada por meio de atividades semelhantes em escolas de Juiz de Fora; e, no formato de workshops em Curitiba e algumas cidades do entorno de Boston, Estados Unidos. Hoje, sabe-se que já ultrapassou o público espectador de 10.000 pessoas (BERMUDEZ et al, 2017; COELHO et al, 2017; GONÇALVES et al, 2016; MADUREIRA et al, 2016; GONÇALVES et al, 2017; SOARES et al, 2017; SOARES et al, 2017; BRITO et al, 2019).

No aprendizado de um comportamento pela experiência direta dos indivíduos, o modo considerado como mais rudimentar de fazê-lo é pelos efeitos positivos ou negativos de suas ações. As capacidades cognitivas habilitam os homens a reforçar ações em seu comportamento, moldando-as pelos resultados previstos. Aprender pelas consequências de suas ações inclui funções como a informativa, na qual os resultados de suas ações servem como guias para futuras ações; motivacional, na qual experiências passadas podem demonstrar que certas ações trazem certos benefícios; de reforço, ou seja, regulando o comportamento que já tinha sido aprendido (BANDURA, 1977). Por outro

INSPIRAÇÃO, COMUNICAÇÃO E EMPATIA COMO CARACTERÍSTICAS ESSENCIAIS 397 À FORMAÇÃO DO MÉDICO HUMANISTA: INOVANDO ATRAVÉS DE UNIVERSIDADES 
DOI: $10.12957 / \mathrm{e}-\mathrm{mosaicos} .2021 .45222$

lado, aprender através de um modelo tem sido visto como o modo no qual o comportamento humano mais tem sido aprendido, ou seja, pela observação dos outros, pelo seu exemplo. Neste caso, foi pelo modelo dos personagens das peças de teatro que os escolares tiveram a oportunidade de conhecer assuntos referentes à fisiologia cardiovascular e a hábitos de vida saudáveis.

Os componentes do processo de aprendizado observacional na análise do aprendizado social são: processo de atenção (no qual a mídia de massa prende nossa atenção, por exemplo); processo de retenção (padrões são representados na memória em forma simbólica); processo de reprodução matriz (convertendo representações simbólicas em ações apropriadas); e processo de motivação (incentivos). Os personagens das peças de teatro do programa "Little Hearts Changing Lives", por meio destes modelos de saúde, contemplaram então os quatro pilares supracitados.

Quando os escolares são envolvidos em atividades em grupo ou participação ativa durante a peça de teatro ou, ainda, durante o reconhecimento de cantigas populares em paródias propostas pela equipe, o objetivo de impactar positivamente o público-alvo torna-se mais próximo. Isto pode ser observado pelos depoimentos de pais e de professores quando os mesmos relatam que as crianças e pré-adolescentes conseguem demonstrar o que aprenderam. E, desta forma, espera-se que o ambiente familiar seja também influenciado.

É imprescindível considerar que a transmissão de mensagens de saúde visando à aquisição de novos padrões de comportamento podem ser transmitidas para as mais variadas audiências por meio de inúmeras linguagens, como o teatro de fantoches, utilizado pelo programa $\mathrm{LHCL}$, trazendo resultados benéficos para toda a sociedade. Entretanto, a consolidação de um alicerce educacional ideal é utópica. Projetos como este são desafiadores, pois não apenas o engajamento dos integrantes do programa é necessário, como também o despertar da curiosidade dos escolares, o interesse dos educadores, da família e da comunidade, esta última envolvendo os serviços de saúde prestados no município em atuação.

As instruções sobre como conduzir o programa tornam os discentes, dos mais variados cursos, aptos a realizarem as atividades junto ao público infantil, devido a sua praticidade e didática. Considera-se esta forma de atuação um aspecto essencial para a formação dos discentes devido a dois fatores igualmente relevante, a saber: primeiro, por aprenderem a lidar com o público de maneira espontânea, especialmente pelo fato de crianças serem receptivas ao novo, favorecendo a criação de vínculo mais facilmente. Assim, pode-se catalisar o preparo do discente para o atendimento ao público num futuro próximo. Segundo, os graduandos colocam à favor da comunidade as bases teóricas ofertadas na universidade.

INSPIRAÇÃO, COMUNICAÇÃO E EMPATIA COMO CARACTERÍSTICAS ESSENCIAIS 398 À FORMAÇÃO DO MÉDICO HUMANISTA: INOVANDO ATRAVÉS DE UNIVERSIDADES 
DOI: $10.12957 / \mathrm{e}-\mathrm{mosaicos} .2021 .45222$

Por outro lado, examinar o contexto em que vive o público-alvo ou o paciente, levando em consideração o espaço em que se insere, vivências, relações familiares e condição social é essencial para o entendimento de situações e patologias em seu sentido mais amplo. Quando discentes são inseridos no ambiente sociocultural do público, a empatia encontra solo fértil para tornar este futuro médico mais solidário em suas ações e condutas.

A disciplina de Fisiologia Cardiovascular da UFJF tem como proposta trazer os conceitos sobre a funcionalidade do sistema cardíaco, não incluindo suas comorbidades. 0 programa LHCL é de legítima importância, para que o próprio discente associe, de forma mais clara, como medidas preventivas podem beneficiar o comportamento fisiológico do organismo como um todo. Abordar assuntos como a alimentação saudável, o estímulo à prática regular de atividade física, dentre outros hábitos comportamentais, é o "core" deste programa.

O curso de Medicina, em seu ciclo básico, aborda profundamente a anatomia e a fisiologia humana em teoria. Questões que tangenciem a compreensão da individualidade de cada ser, geralmente ocorrerá quando, em períodos futuros, o aluno tiver contato com ambulatórios e redes de atenção à saúde em seus vários níveis de complexidade (primário, secundário e terciário). Entretanto, Projetos de Extensão permitem ao aluno do curso de Medicina alçar novos olhares e outros voos, rompendo precocemente barreiras entre a academia e a comunidade, em direção ao seu objetivo final: a consolidação do conhecimento teórico por meio da vivência para a promoção da saúde e do bem-estar.

Ações como as propostas pelo programa LHCL possibilitam, então, o desenvolvimento de habilidades como a comunicação. (NATÁRIO; BATISTA, 2018). A ação extensionista, portanto, permite experimentações e vivências, sendo assim, de fundamental importância para a consolidação do aluno como profissional humanizado (ALMEIDA et al., 2016).

\section{Depoimentos}

"Sendo o programa LHCL baseado em ações educativas e saúde para um públicoalvo específico, pode-se dizer que este projeto também tem um importante papel na afeição do discente pela dinâmica da Medicina da Família, realidade da saúde brasileira e de extrema importância para a população. O aluno se sente confortável e estimulado a conversar sobre prevenção de fatores de risco, o que é crucial na formação do médico, utilizando novas formas de interação e linguagens com diversos públicos. Neste programa, o teatro de fantoches é o meio de comunicação para que sejam transmitidos e/ou reforçados conhecimentos sobre hábitos saudáveis." AFAS, aluna do curso de Medicina, participante do programa LHCL de 2015 a 2016.

INSPIRAÇÃO, COMUNICAÇÃO E EMPATIA COMO CARACTERÍSTICAS ESSENCIAIS 399 À FORMAÇÃO DO MÉDICO HUMANISTA: INOVANDO ATRAVÉS DE UNIVERSIDADES 
DOI: $10.12957 / \mathrm{e}-\mathrm{mosaicos} .2021 .45222$

\section{CONSIDERAÇõES FINAIS}

O programa "Little Hearts Changing Lives" tem contribuído de forma proativa para inspirar e instigar o espírito criativo e inovador de discentes de cursos de Medicina, dentre outros. Possibilita, desta forma, que os discentes não se limitem ao ambiente puramente teórico, mas vivenciem a rotina de comunidades distintas e tenham a oportunidade de explorar habilidades próprias como o protagonismo, a comunicação, a criatividade, a empatia, a colaboração e a elaboração de conteúdo para o público leigo e produção de textos científicos. Desta forma, permite-se que os discentes tenham a liberdade de ação, além dos muros da universidade, tornando-os cidadãos mais atuantes na realidade.

\section{REFERÊNCIAS}

ALMEIDA, L. E. de; PEREIRA, M. N.; OLIVEIRA, V. de. Governador Valadares (MG) em Extensão: Interfaces para a Dinamização e Instrumentalização do Cenário Extensionista em um Campus Recém-Implantado. Rev. bras. educ. med., Rio de Janeiro, v. 40, n. 4, p. 743-750, dez. 2016.

BANDURA, A. Social Cognitive Theory of Mass Communication. In: Media Effects: advances in theory and research. USA: Lawrence Erlbaum Associates, Hillsdale, New Jersey, Hove, UK, 1994.

BANDURA, A. Social Learning Theory. New Jersey: PRENTICE-HALL, INC. Englewood Cliffs, 1977.

BERMUDES, B.E.B.V., ALVIM R.O., SOARES F.M., GONÇALVES A.B.C., TIZZOT E.L.A., ULBRICH A., MOURÃO C.A., JR. \& OLIVEIRA C.M. The Baependi Little Heart Study: Strategies in Child Education Related to Cardiometabolic Risk Factors for Reducing Morbidity and Mortality in a Developing Country. Endocrinology \& Metabolism International Journal, v. 5, n. 5, p. 1-4, 2017.

BRITO, R.S., BERMUDEZ B.E.B.V., TIZZOT, E.L.A., SIQUEIRA C.E, DE OLIVEIRA C.M. Construção de projetos lúdicos em saúde através da capacitação de educadores em Framingham, Massachusetts, Estados Unidos. Revista Brasileira de Extensão Universitária, v.10, n.2, p.87-93, 2019.

COELHO, P. D., QUEIROZ, H. K. G., PEREIRA, A. P. S., VIEIRA, P. P., DE OLIVEIRA ALVIM, R., JÚNIOR, C. A. M., \& OLIVEIRA, C. M. Práticas educativas relacionadas à saúde para crianças de 4 a 10 anos. Projeto Coraçõezinhos apaixonados: um relato de experiência. Extensão em Foco, v. 1, n. 13, 2017. 
DOI: $10.12957 / \mathrm{e}-\mathrm{mosaicos} .2021 .45222$

GONÇALVES, A. B. C., F. M. SOARES, R. DE OLIVEIRA ALVIM, C. A. M. JÚNIOR AND C. M. J. E. R. C. D. E. DE OLIVEIRA. Dengue, Zika e Chikungunya: o combate começa nas escolas. Experiência. Revista Científica de Extensão. v. 2, n. 2, 2016.

GONÇALVES, A. B. C., F. M. SOARES, R. DE OLIVEIRA ALVIM, C. A. M. JÚNIOR AND C. M. J. I.-R. D. E. D. U. OLIVEIRA. Análise de uma intervenção comunitária para crianças: A promoção da saúde do Idoso e o projeto coraçõezinhos de Baependi. Revista Interfaces UFMG, v. 5 n. 2, p. 211229, 2017.

KRUG, R.R., VIEIRA M.S., MACIEL M.V., ERDMANN T.R., VIEIRA F.C., KOCH M.C., GROSSEMAN S. O "Bê-Á-Bá" da Aprendizagem Baseada em Equipe. Rev. bras. educ. med., Rio de Janeiro, v. 40, n. 4, p. 602-610, Dec. 2016.

MADUREIRA M.F., PEIXOTO L.M.M., HARAMOTO H., SIBREIRA, N.P., PEREIRA N.S., FERREIRA, R.N., PEREIRA L.S., ALVIM R.O., MOURÃO C.A., JR. \& OLIVEIRA C.M. (2016). Coraçõezinhos de Baependi and health education through participatory methodology: experience report. Revista Conexão UEPG, v. 12, n. 3, p. 400-411, 2016.

NATÁRIO EG, BATISTA MD. Projeto Santos Jovem Doutor-Desdobramentos para uma Aprendizagem significativa em Saúde na Educação Básica. e-Mosaicos. v.7, n.15, p. 69-88, 2018.

OLIVEIRA, M.P.R. de. Formação e qualificação dos profissionais de saúde: fatores associados à qualidade dos serviços de atenção primária à saúde. 2014. 174 f. Dissertação (Mestrado em Ensino na Saúde) - Universidade Federal de Goiás, Goiânia, 2014.

PEIXOTO, M.A.P. Gostar e Compartilhar - Um Novo Tempo para a Educação Médica. Rev. bras. educ. med., Rio de Janeiro, v. 41, n. 3, p. 361-363, Sept. 2017.

RIOS, L.E. ABC das Teorias de Mudança de Comportamento: Resenha crítica. Rev. bras. educ. med., Rio de Janeiro, v. 41, n. 2, p. 356-358, June 2017.

RUSK N., RESNICK, M., COOKE, S. Origins and guiding principles of the computer clubhouse. The computer clubhouse: Constructionism and creativity in youth communities. p. 17-25, 2009.

SILVA JÚNIOR, G.B. MEDEIROS M.A., OLIVEIRA J.G., CATRIB A.M., JARDIM M.H. Team-Based Learning: Successful Experience in a Public Health Graduate Program. Rev. bras. educ. med., Rio de Janeiro, v. 41, n. 3, p. 397-401, Sept. 2017.

SILVA A.T.C., MEDEIROS JUNIOR M.E.D., FONTÃO P.D.N., SALETTI FILHO H.C., VITAL JUNIOR P.F., BOURGET M.M.M., RIOS I.C. Medicina de Família do Primeiro ao Sexto Ano da Graduação Médica: Considerações sobre uma Proposta Educacional de Integração Curricular EscolaServiço. Rev. bras. educ. med., Rio de Janeiro, v. 41, n. 2, p. 336-345, June 2017. 
DOI: $10.12957 / \mathrm{e}-\mathrm{mosaicos} .2021 .45222$

SOARES F.M., GONÇALVES A.B.C., ALVIM R.O., MOURÃO C.A., JR. \& OLIVEIRA C.M. Conscientização infantil: abordagem lúdica sobre utilização de recursos naturais. Revista Ciência em Extensão, v. 13, n. 3, p. 87-92, 2017.

SOARES F.M., GONÇALVES A.B.C., ALVIM R.O., MOURÃO C.A., JR. \& OLIVEIRA C.M. Método educacional infantil na prevenção e combate à dengue, zika vírus e Chikungunya. Revista Conexão em Foco, v. 1, n. 13, p. 55-63, 2017.

SOMBRA NETO, L.L. SILVA V.L., LIMA C.D., MOURA H.T., GONÇALVES A.L., PIRES A.P., FERNANDES V.G. Habilidade de Comunicação da Má Notícia: o Estudante de Medicina Está Preparado? Rev. bras. educ. med., Rio de Janeiro, v. 41, n. 2, p. 260-268, June 2017.

\section{AgRADECIMENTOS}

Agradecimento especial aos alunos dos cursos de Medicina, Nutrição e Enfermagem que mantiveram o projeto em pleno funcionamento e a todo vapor por 2 anos ininterruptos. Muito obrigada a cada uma de vocês, meninas!

Recebido em 12 de setembro de 2019

Aceito em 16 de novembro de 2020



A e-Mosaicos Revista Multidisciplinar de Ensino, Pesquisa, Extensão e Cultura do Instituto de Aplicação Fernando Rodrigues da Silveira (CAp-UERJ) está disponibilizada sob uma Licença Creative Commons - Atribuição - NãoComercial 4.0 Internacional.

Os direitos autorais de todos os trabalhos publicados na revista pertencem ao(s) seu(s) autor(es) e coautor(es), com o direito de primeira publicação cedido à e-Mosaicos.

Os artigos publicados são de acesso público, de uso gratuito, com atribuição de autoria obrigatória, para aplicações de finalidade educacional e não-comercial, de acordo com o modelo de licenciamento Creative Commons adotado pela revista. 